(Aus dem physiologischen Laboratorium der Universität Halle.)

\title{
Versuche über einige physiologische Wirkungen des Natriumcarbonates.
}

\author{
Von
}

K. Schoenlein. cand. med.

Hierzu Tafel II.

Von Herrn Prof. Bernstein aufgefordert, unternahm ich es, die Einwirkung des kohlensauren Natrons auf Rhythmus und Form der Herzschläge zu studieren. Die Untersuchung bot practisches Interesse, da sich ausser Traube ${ }^{1}$ ), und dieser nur gelegentlich, von den Physiologen Niemand um die Frage gektimmert zu haben scheint, wie das kohlensaure Natron auf den Organismus wirkt, und dasselbe doch so vielfältig zu manometrischen Versuchen verwendet wird.

Die Untersuchung ist bis jetzt nur an Fröschen vorgenommen und muss noch auf Warmbliuter ausgedehnt werden. Beobachtet wurden diastolischer Ventrikelstillstand nach geringeren Gaben, nach einiger Zeit wieder verschwindend, bei grösseren Dosen eine nachfolgende langsame und andauernde Contraction des Ventrikels, welche nicht immer spontan aufhörte, and endlieh beim nicht curaresirten Thier Allgemeinkrämpfe. Veränderungen im Rhythmus wurden eclatant nur bei manometrischen Versuchen, und auch da nicht immer beobachtet.

1) Traube ges. Beitr. Bd. I. p. 248, 372, 384. 
Nachdem der Frosch in der Rückenlage auf einem Froschbrette befestigt war, wurde das Herz in bekannter Weise durch Wegnahme des Proc. xyphoideus und einen median von unten nach oben verlaufenden Scheerenschnitt, der den Schultergürtel trennte, freigelegt. In die mittlere Bauchvene wurde nach dem Herzen zu, da wo sie sich unterhalb des Proc. xyphoideus von der vorderen Bauchwand abhebt, eine feine Glascaniüle eingebunden, welche durch ein kurzes Gummiröhrchen mit einer graduirten Spritze verbunden war, and das peripherisehe Ende der Vene abgeschnüirt. Als Injectionsflüssigkeit diente 10\% ige oder 5\% ige Lösung von Natriumearbonat in destillirtem Wasser. Einige Male wurde anch reine Natronlauge oder kohlensaures Ammoniak in Lösungen von ungefähr gleicher Concentration verwendet. Die Injectionsmenge schwankte zwischen 0,1 bis $1 \mathrm{ccm}$.

Der Versuch wurde so angestellt, dass zuerst nach dem Sekundenzeiger einer Tasehenuhr die Zahl der Herzcontractionen in einer Minute bestimmt wurde; wenn dieselbe einige Zeit gleich geblieben war, wurde der Stempel der mit der Lösung gefüllten Spritze langsam um die beabsichtigte Anzahl von Theilstrichen eingetrieben. Die Injection geschah nach vollendeter letzter Zählung. Nach derselben wurde die Zählung zunächst sistirt, um den anderweitigen Erfolg zu beobachten, und dieselbe meist erst nach einigen Minuten wieder aufgenommen. Wurde es für wünschenswerth gehalten, die Zählung nicht zu unterbrechen, so richtete sie sich, wenn der Ventrikel durch die Injection zum Stillstand kam, nach den Vorhofspulsen. Um während des Ventrikelstillstandes, und namentlich während des Contractionszustandes das Herz von der Natriumearbonatlösung zu befreien, wurde 0,75\% ige Kochsalzlösung nachgespült. Thre Menge war gross, etwa $10 \mathrm{ccm}$ oder mehr; der starke Druck, welcher sich in Folge der grossen injicirten Flüssigkeitsmenge im Gefässsystem einstellte, wurde durch Aufschneiden der Lymphsäcke an den Unterschenkeln und Oeffnen irgend eines Gefässes beseitigt oder ging in Folge der wieder eintretenden Blutung, welche durch das Natriumcarbonat gesetzt wurde, von selbst wieder vorüber. Die zum Ausspülen verwendete Flïssigkeit musste mit einiger Kraft eingetrieben werden, wodurch der Sinus venosus und die Vorhöfe prall und stark gefült wurden.

Der diastolische Ventrikelstillstand trat gleich beim ersten Versuche ein. Derselbe folgt: 
Versuch Nro. I am 17./1. 78.

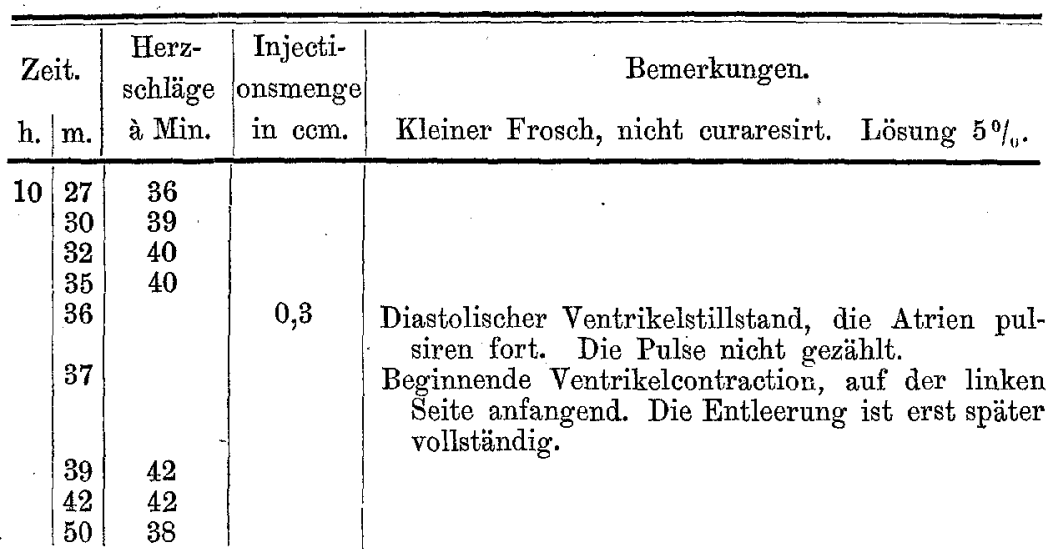

Der beobachtete Erfolg des diastolischen Ventrikelstillstandes führte zu der Frage, ob derselbe von einer Reizung hemmender Centren; sei es im verlängerten Mark, sei es im Herzen selbst, abzuleiten sei. Das verlängerte Mark wurde desshalb bei einigen Versuchsthieren zerstört, indem zwischen Hinterhaupt und Atlas ein glïhender Draht eingesenkt, und mit demselben ein wenig: nach oben und unten in das Gehirn und Rïckenmark eingegangen wurde. Der Erfolg, blieb ungeändert; wie der nachstehend angeführte Versuch zeigt.

Versuch Nro. II am 19./1. 78.

\begin{tabular}{|c|c|c|c|c|}
\hline \multicolumn{2}{|c|}{ Zeit. } & Herz* & Injecti- & \\
\hline h. & $\mathrm{m}$. & à Min. & in $\mathrm{ccm}$. & candens zerstört, nicht curaresirt. Lösung $5 \%$. \\
\hline 10 & $\begin{array}{l}55 \\
60\end{array}$ & $\begin{array}{l}38 \\
40\end{array}$ & & \\
\hline 11 & 2 & 40 & & \\
\hline & $\begin{array}{l}4 \\
5\end{array}$ & & 0,1 & Ohne Wirkung. \\
\hline & 7 & 40 & & \\
\hline & 10 & 40 & & \\
\hline & 13 & & 0,3 & $\begin{array}{l}\text { Diastolischer Stillstand der rechten Hälfte des } \\
\text { Ventrikels. Die linke Hälfte pulsirt noch, doch } \\
\text { contrahirt sie sich nicht vollständig. Die Atrien } \\
\text { pulsiren weiter. }\end{array}$ \\
\hline & 15 & 40 & & $\begin{array}{l}\text { Der ganze Ventrikel pulsirt. Tetanus des Ver- } \\
\text { suchsthieres. }\end{array}$ \\
\hline & $\begin{array}{l}20 \\
21\end{array}$ & 40 & 0,1 & $\begin{array}{l}\text { Die rechte Hälfte des Ventrikels während der Sy- } \\
\text { stole dunkler als die linke. Diastolischer Still- } \\
\text { stand nicht vorhanden. }\end{array}$ \\
\hline
\end{tabular}


Versuche über einige physiologische Wirkungen des Natriumcarbonates. 29

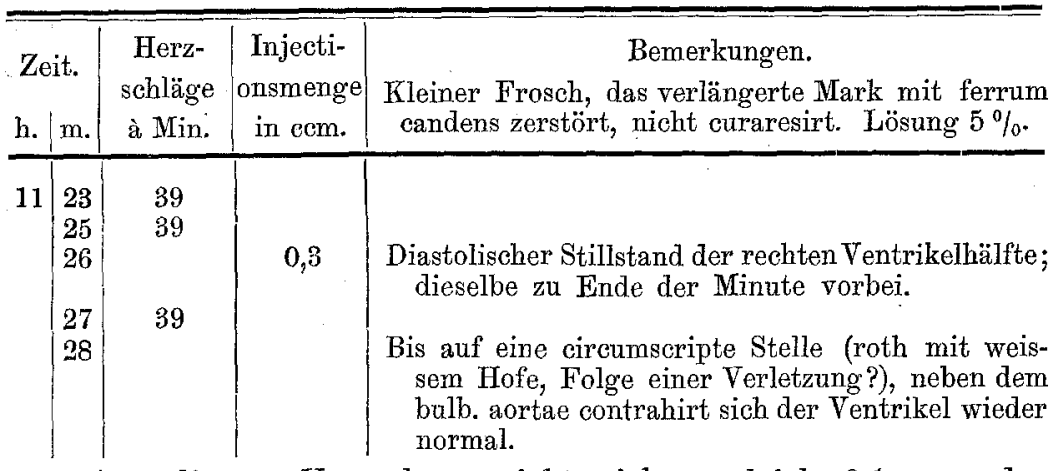

Aus diesem Versuche ergiebt sich zugleich $0,1 \mathrm{ccm}$ der $5 \%$ igen Lösung als ungefähr geringste Menge, welche noch eine Wirkung hervorzubringen vermag, denn die während der Systole rothe Farbe der rechten Ventrikelhälfte ist daraus zu erklären, dass der Ventrikel an dieser Seite in contrahirtem Zustande noch etwas Blut enthält, sich also nicht vollständig zusammenzuziehen vermag.

Die Krämpfe, welche bei diesem Versuche auftreten und bei den meisten Versuchen am uncuraresirten Thiere wiedergefunden wurden, haben, wie einige hierauf gerichtete Versuche zeigten, ihren Ursprung im Rückenmark. Da sie bei curaresirten Thieren ausblieben, war zunächst die Möglichkeit ausgeschlossen, dass durch das in den Kreislauf gekommene kohlensaure Natron die Substanz der willkührlichen Muskeln selbst erregt würde. Die Durchschneidung des Nervus ischiadicus in der Beckenhöhle und am Oberschenkel zeigte, dass auch die Nervenstämme in ihrem Verlauf vom Rückenmark zu den Muskeln nicht von der Injection betroffen wurden, denn der Tetanus blieb an der so operirten Extremität aus. Er trat ein, so lange das Rückenmark intact war, und sein Ursprung kann, wie der vorstehende Versuch zugleich zeigt, nicht in die Medulla oblongata verlegt werden. Wurde das Riuckenmark mit einer Sonde ganz zerstört, so traten höchstens hier und da einzelne Zuckungen der Muskeln ein, reichte die Zerstörung des Rückenmarks nicht durch den ganzen Wirbelcanal hindurch, so traten heftige Bewegungen oder Tetanus in den unteren Extremitäten auf. Ob die beobachteten Erscheinungen reflectorische oder durch Erregung motorischer Nerven allein zu Stande gebrachte seien, wurde nicht entschieden. Einseitige Durchschneidung der hinteren Rückenmarkswurzeln änderte nichts. In der Chloroformnarkose blieb der Tetanus aus, war aber nach Er- 
In einem weiteren Versuche wurden, um Einwirkungen des verlängerten Markes noch sicherer auszuschliessen, beide Vagi abgebunden, nachdem vorher das verlängerte Mark zerstört worden war, beides ohne den Erfolg der Injection zu ändern. Dabei wurden auch grössere Dosen kohlensauren Natrons gegeben, als frihher, um den Erfolg anschaulicher zu machen. Der Versuch folgt:

Versuch Nro. III am 24./1. 78.

\begin{tabular}{|c|c|c|c|}
\hline h. $\mathrm{m}$. & $\begin{array}{l}\text { Hèrz- } \\
\text { schläge } \\
\text { à Min. }\end{array}$ & $\begin{array}{c}\text { Injecti- } \\
\text { onsmenge } \\
\text { in ccm. }\end{array}$ & $\begin{array}{l}\text { Bemerkungen. } \\
\text { Kleiner Frosch, nicht curaresirt, das verlängerte } \\
\text { Mark mit ferrum candens zerstört. Kohlensaures } \\
\text { Natron } 10 \% \text { ig. }\end{array}$ \\
\hline $\begin{array}{l}23 \\
26 \\
27 \\
30 \\
45\end{array}$ & $\begin{array}{l}46 \\
46\end{array}$ & 0,2 & $\begin{array}{l}\text { Diastolischer Stillstand des ganzen Ventrikels, } \\
\text { später anhaltende Contraction der rechten Seite. } \\
\text { Die Contraction rechterseits bis auf einige Stellen } \\
\text { (verletzt?) verschwunden. } \\
\text { Die beiden Vagi abgebunden. Dabei links die } \\
\text { Carotis zerrissen, starke Blutung. } \\
\text { Diastolischer Stillstand des ganzen Ventrikels. } \\
\text { Eine langsam eintretende und lange anhaltende } \\
\text { Contraction folgt nach. Die Vorhöfe machen } \\
\text { in der Minute } 40 \text { Pulse. Nach dem Ausschneiden } \\
\text { und Ausspülen mit 0,75\% \%iger Kochsalzlösung } \\
\text { pulsirt der Ventrikel wieder. }\end{array}$ \\
\hline
\end{tabular}

Nachdem sich auf diese Weise ergeben hatte, dass eine durch die Injection gesetzte Reizung des Hemmungscentrums im verlängerten Mark nicht vorhanden war, wurden in einigen weiteren Versuchen die Hemmungsnerven im Herzen selbst durch Gifte gelähmt. Als solche wurden Curare and Atropin verwendet. Es folgt ein solcher Versuch mit Curare. Bei demselben wurde auch mehr als sonst auf die Zahl der Herzschläge in der Minute geachtet. Während des Ventrikelstillstandes wurden die Vorhofspulse weiter gezählt. 
Versuche über einige physiologische Wirkungen des Natriumcarbonates. 31

Versuch Nr. V am 29./1. 78.

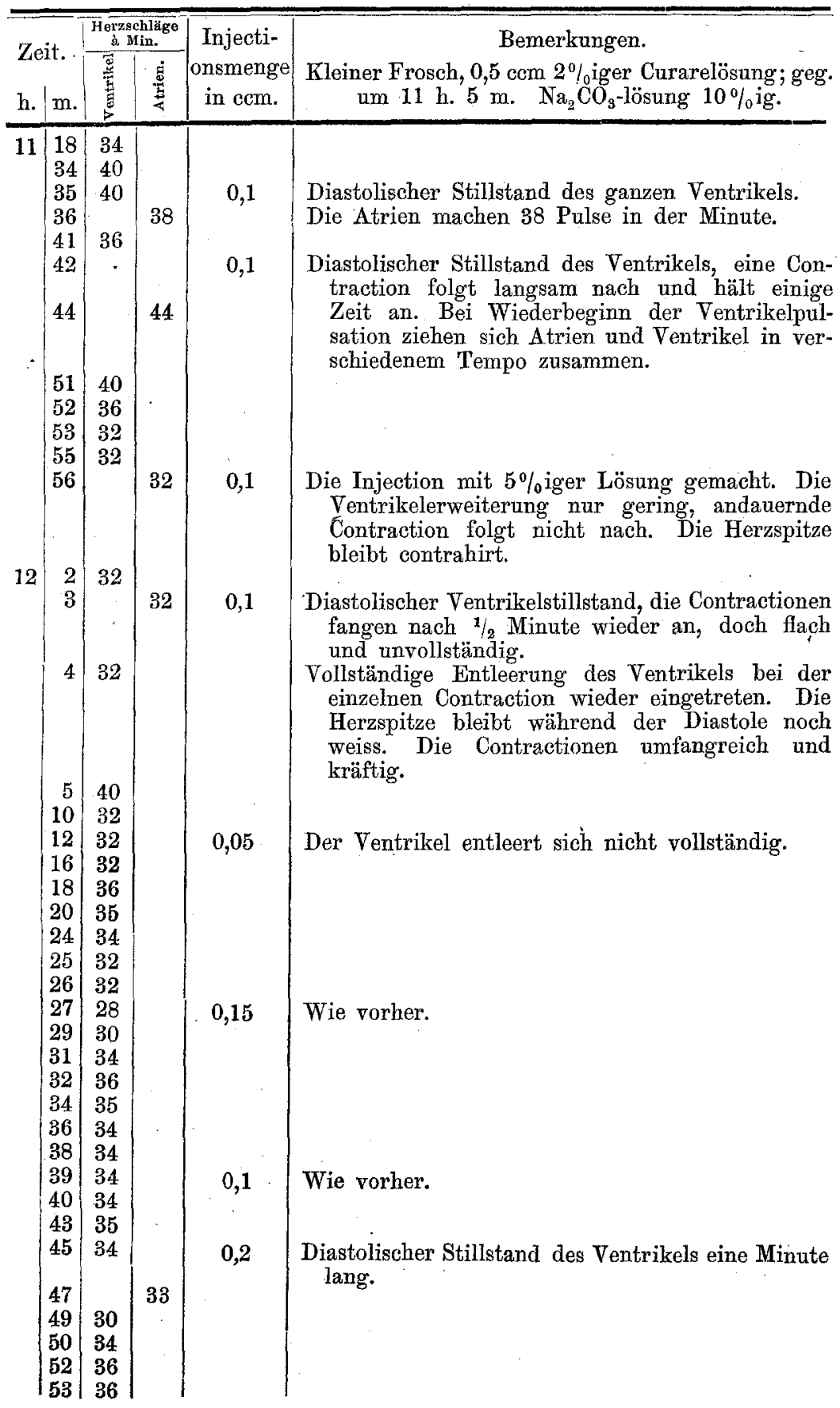


Die Unterschiede im Rhythmus der Schläge sind, wie man sieht, sehr gering. Im Ganzen zeigt sich eine Verlangsamung gegen Ende des Versuches, die nach jeder einzelnen Injection durch eine kurze Beschleunigung, unterbrochen wird.

Die bei der zweiten Injection in diesem Versuche bemerkte Verschiedenheit im Tempo der Atrien und Ventrikelcontraction verdient besonders bemerkt zu werden. Es wurde in dieser Zeit der Ventrikel durch mehrere Vorhofscontractionen erst stark gefüllt, ehe er sich zusammenzog. Dabei kam es vor, dass sich Ventrikel und Atrien vollständig gleichzeitig zusammenzogen. Die Mengen der durch die einzelnen Ventrikelcontractionen fortgeschafften Flüssigkeiten waren, so viel sich aus der jedesmaligen Grösse des sich contrahirenden Ventrikels ersehen liess, nicht immer dieselben, sondern abwechselnd grösser und kleiner. Auf eine bestimmte Reihenfolge dieser Abwechselung war nicht besonders geachtet worden.

Bei den nun vorgenommenen Versuchen mit Atropin wurde zugleich durch Reizung mit dem Schlitteninductorimm die Atropinwirkung festgestellt. Die als Electroden dienenden Reiznadeln wurden rechts und links auf der Rïckseite des Halses neben dem Atlas eingestochen. Ich führe folgenden Versuch an.

Versuch Nro. VII am 1./2. 78.

\begin{tabular}{|c|c|c|c|c|c|}
\hline \multicolumn{2}{|c|}{ Zeit. } & \multicolumn{2}{|c|}{$\begin{array}{c}\text { Herzaschläge } \\
\text { à Min. }\end{array}$} & \multirow{2}{*}{$\left|\begin{array}{c}\text { Injecti- } \\
\text { onsmenge } \\
\text { in ccm. }\end{array}\right|$} & \multirow{2}{*}{$\begin{array}{l}\text { Bemerkungen. } \\
\text { Grosser Frosch, curaresirt. Atropin 2,4 mgr } \\
\text { subcutan. } \mathrm{Na}_{2} \mathrm{CO}_{3} \text {-lösung } 5^{\circ} \% \text { ig. }\end{array}$} \\
\hline h. & $\mathrm{m}$. & 焉 & 害 & & \\
\hline \multirow[t]{2}{*}{11} & 14 & 32 & \multirow{7}{*}{32} & \multirow{6}{*}{0,3} & \multirow{4}{*}{ 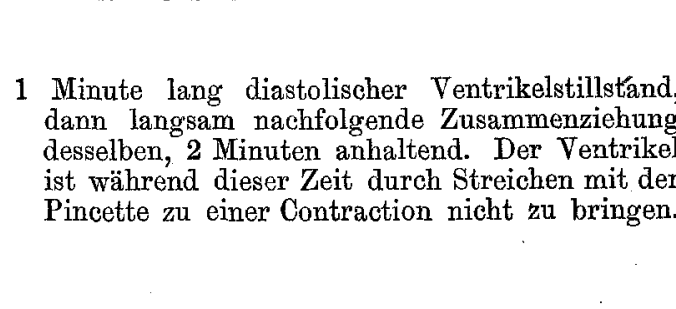 } \\
\hline & $\begin{array}{l}39 \\
40\end{array}$ & 33 & & & \\
\hline 12 & 45 & $\begin{array}{l}40 \\
36\end{array}$ & & & \\
\hline & 3 & 36 & & & \\
\hline & 7 & 34 & & & 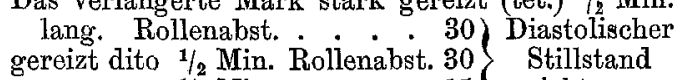 \\
\hline & $\begin{array}{r}8 \\
11 \\
23\end{array}$ & $\begin{array}{l}33 \\
31 \\
30\end{array}$ & & & $" \quad 1 / 2 \mathrm{Min} . \quad$ handen, Atropin wirksam! \\
\hline & 25 & 31 & & 0,3 & $\begin{array}{l}\text { Diastolischer Ventrikelstillstand } 2 \text { Minuten, dann } \\
\text { eine langsame Contraction, in welcher der Ven- } \\
\text { trikel } 11 / 2 \text { Minute verbleibt. }\end{array}$ \\
\hline
\end{tabular}


Versuche über einige physiologische Wirkungen des Natriumcarbonates. 33

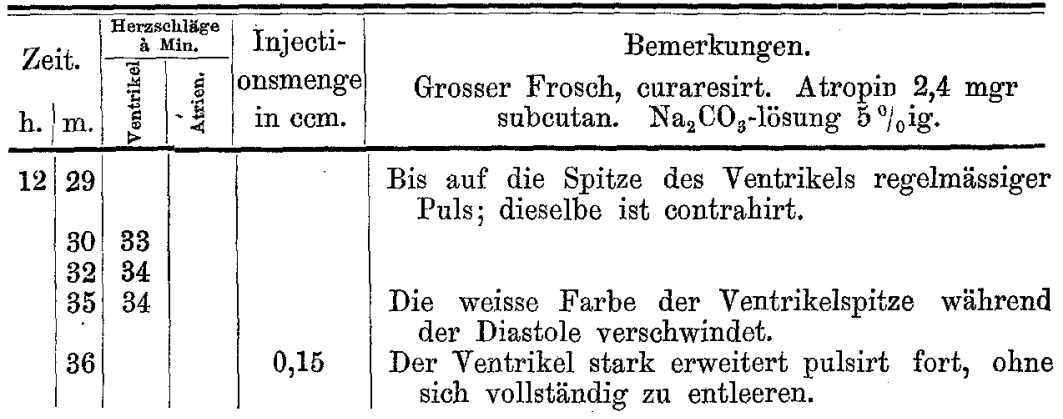

Diese Versuchsweise wurde noch dahin variirt, dass die Atropininjection erst dann geschah, wenn vorher durch Reizung: des verlängerten Markes Herzstillstand erzeugt, und somit dargethan war, dass der electrische Reiz wirksam sei. Die Atropininjection wurde daranf in die mittlere Banchvene gemacht, und nach einiger Zeit versucht, ob durch Reizung mit Strömen von gleicher oder grösserer Intensität noch Herzstillstand zu erreichen war; sobald derselbe ausblieb, die Atropinwirkung also manifest war, wurde der $\mathrm{Na}_{2} \mathrm{CO}_{3}$-Versuch in der gewohnten Weise vorgenommen. Da der Erfolg derselbe blieb, ist es nicht nöthig, diese Versuche anzuführen.

Um zu erfahren, ob das Natriumearbonat als solches, oder durch seine alkalische Reaction wirke, wurden Versuche gleicher Art mit anderen alkalisch reagirenden Substanzen gemacht, nämlich Natronlauge, kohlensaurem Ammoniak und Barytwasser. Auch wurde in einzelnen Versuchen zwischen kohlensaurem Natron und Kochsalz in 5\% iger Lösung abgewechselt, um zu sehen, ob eine Lösung neutralen Alkalisalzes von gleicher Concentration dieselben oder ähnliche Wirkungen zeige, wie die alkalisch reagirenden Salze. Nach Kochsalzinjection trat keine Veränderung der Herzschläge ein. Die Wirkungen der anderen vorgenannten Substanzen waren denen des kohlensauren Natrons sehr ähnlich, so weit die oberflächliche Betrachtung dies zeigte. Am intensivsten, stärker noch als die des kohlensauren Natrons, war die Wirkung des kohlensauren Ammoniaks.

Die in dieser Versuchsreihe beobachteten Erscheinungen sollten nun auch graphiseh wiedergegeben werden. Es wurde dazu das Dreiwegehahnsystem von Bowditsch 1) in Verbindung

1) Abgebildet i. Cyon Experimentalphys. Taf. LIII. 3. Beschr. p. 556 E. Pflüger, Archiv f. Physiologie, Bd, XVIII. 
mit dem Froschmanometer angewendet. In die linke Aorta des vorher curaresirten Versuchsthieres wurde eine Glascanüle, dem Herzen zugewendet, eingeführt und dieselbe durch einen kurzen Gummischlanch mit dem, dem Manometer näheren, unteren freien Röhrenende verbunden. Zwischen Gummischlauch und Manometer befand sich so nur ein Hahn. Zur Fuillung für das Hahnsystem und den Gummischlauch durfte natürlich nicht Natriumcarbonat, als der zu untersuchende Körper, genommen werden. Um aber die störende Gerinnung des Blutes zu vermeiden, wurde das ganze Thier mit Kochsalzlösung so lange durchgewaschen, bis das Blut hinlänglich verdünnt war, um nicht mehr gerinnen zu können. $\mathrm{Zu}$ diesem Zwecke wurde in die mittlere Bauchvene, dem Herzen zu, mittelst Glascanüle und Gummischlauch eine etwa $10 \mathrm{ecm}$ haltende Spritze eingefithrt, und aus der angeschnittenen linken Aorta das Blut ablaufen gelassen. War dureh die Verblutung der Gesammtdruck sehr gesunken und der Umfang der Herzcontractionen verkleinert, so wurde aus der Spritze langsam Kocbsalzlösung nachgetrieben und die linke Aorta eine kleine Weile abgeklemmt, um die Lösung im Körper umzutreiben und sie sich mit dem Blute des Thieres gehörig mischen zu lassen. Dann wurde die Klemme von der linken Aorta weggenommen, und. das ganze Verfahren mehrmals wiederholt, bis die Flüssigkeit aus der nun in die Aorta eingeführten Canïle klar und höchstens hellgelb gefärbt abfloss. Diese Operation nahm etwa 10 Minuten in Anspruch. Nachdem sie vollendet war, wurde die Canüle, wie schon vorher erwähnt war, mit dem Manometer verbunden und die jetzt natürlich sehr geringe Spannung im Gefässsystem durch einen kleinen Nachschub von Kochsalzlösung bis zu 8 oder $10 \mathrm{~mm}$ Quecksilberdruck erhöht. Nachdem nun einige normale Pulse verzeichnet waren, wurde eine Injection gemacht, und zwar meist etwas mehr als früher gegeben, da die durch Abbindung der einen Aorta gesetzte Kreislaufsstörung das Natriumcarbonat nicht so schnell zur Cirealation gelangen liess als in den früheren Versuchen. Der Moment der Injection wurde auf den Curven notirt.

Bei sämmtlichen Curven manifestirt sich die Injection ausnahmslos durch Absinken des Druckes und Aufhören der Pulsationen, oder mindestens durch bedeutende Verflachung: der einzelnen Elevationen. Verlangsamung der einzelnen Pulse mit gleichzeitiger Vergrösserung des Umfangs und der Höhe der einzelnen Pulscurve 
Versuche über einige physiologische Wirkungen ḋes Natriumearbonates. $\mathbf{3}$

trat nicht immer gleich nach der Injection ein, sondern öfter beträchtlich später, blieb jedoch nicht aus. Ist sie einmal eingetreten, so bleibt sie bis zu Ende des Versuchs, und dann zeigen sich eigenthïmliche Formen des absteigenden Theils der einzelnen Curve ${ }^{1}$ ). Auf denselben sind ein, zwei, oder auch mehrere Erhebungen aufgesetzt. Wo diese vorhanden sind, ist die einzelne Erhebung länger als die vorangehenden einfachen Elevationen, doch kürzer als zwei bei denen mit einer aufgesetzten Welle, kürzer als drei oder mehr bei denen mit drei oder mehr aufgesetzten Wellen. Ferner wurden im regelmässigen Rhythmus abwechselnde grosse und kleine Pulsationen notirt. Beide Pulsarten bleiben immer einige Zeit, doch nicht permanent, sondern verschwinden wieder.

Es war nun noch zu untersuchen, wie sich der Ventrikel allein gegen die Injectionsflüssigkeit verhielt. Zu diesem Zwecke wurde er durch Abquetschen von den nervösen Elementen des Herzens isolirt. Die Operation selbst wurde vollzogen, indem er zwischen die glattrandigen Branchen einer der Atrioventriculargrenze parallel und unterhalb derselben gelegten Pincette gefasst, and dieselben im Moment der Diastole mit Daumen und Zeigefinger beider Hände scharf gegeneinander gedriickt wurden ${ }^{2}$ ). Auf diese Weise konnte der Ventrikel noch bequem mit der Versuchsflüssigkeit gefüllt werden. War dieser Handgriff richtig gemacht, so zeigte der abgequetschte Ventrikel, wenn vorher keine Natriumcarbonatinjection gemacht war, auf jeden einzelnen Reiz nur eine einzelne Contraction, und verweilte sonst in Diastole, während der an der Atrioventriculargrenze sitzende Rest noch fortpulsirte. Die Abquetschung wurde vor oder nach der Natriumearbonatinjection vorgenommen. Im ersten Falle erweiterte sich die abgequetschte Parthie noch unter dem Einfluss der Lösung, im zweiten war sie schon erweitert, in beiden Fällen blieb sie gegen mechanischen Reiz unerregbar.

Um auch für den isolirten Ventrikel die graphische Methode verwenden zu können, verfertigte ich mix eine Herzkanüle nach Art der Kronecker'schen Doppelcanüle. Das mit dem Sinus venosus und den Vorhöfen ausgeschnittene Herz wurde mit Stecknadeln auf einen Kork befestigt, die Canüle durch den Sinus venosus und rechten Vorhof hindureh in den Ventrikel vorgeschoben und letz-

1) Vergl. Traube, ges. Beitr. Bd. I. p. 372.

2) Siehe: Bernstein. Medic. Centralblatt. 1876. ю. 385 . 
terer unterhalb der Atrioventrikulargrenze zwischen den beiden Knöpfen der Canüle festgebunden, nachdem er vorher durch Festbinden der Vorhöfe in der gewünschten Lage auf der Canïle fixirt war. Verletzungen des Ventrikels wurden sorgfältig vermieden. Die Unterbindung wurde weit genug unten gemacht, so dass auf Reiz nur eine Einzelcontraction erfolgte. An die beiden Röhrenenden der schon vorher mit defibrinirtem und auf das Doppelte seines Volums mit 0,75\% iger Kochsalzlösung verdünnten Rinderblut gefülltten Canüle wurden mit demselben Blut gefüllte Gummiröhrchen angesetzt und die Canüle durch letztere mit dem Hahnsystem von $B$ owditseh verbunden, nachdem dieses gleichfalls mit verdünntem Blute gefüllt, und alle Luftblasen sorgfältig entfernt waren. Dann wurde unter das Herz ein mit Blut gefiillter Trichter gesetzt, in dessen nach oben umgebogenen und mit Quecksilber gefullten Hals ein Kupferdraht als eines Ende der secundären Rolle eines Schlittenapparates eintauchte, während das andere Ende derselben an der Herzcanüle befestigt war. Tauchte das Herz in das Blut des Trichters ein, so konnte der electrische Strom der secundären Rolle durch dasselbe geleitet werden. In den secundären Kreis war noch ein Schlïssel als Nebenschliessung, und in den primären eine Wippe eingeschaltet, um einzelne Schläge nach Belieben durch das Herz schîcken zu können. Nachdem nun das Herz an den Hahnapparat angesetzt und alles andere vorbereitet war, wurden alle Verbindungen ausser der vom Herz zum Manometer gehenden abgesperrt und eine einzelne Zuckung mit minimalem Reiz, so dass der Oeffnungsschlag eben wirkte, verzeichnet. Darauf wurde in die an dem Apparat befestigste Druckflasche verdinntes Blut, mit der gleichen oder doppelten Menge 5\% iger Natriumcarbonatlösung versetzt, eingegossen und abermals gereizt. Der jetzige Reiz wirkte entweder, auch wenn or nun sehr verstärkt wurde, gar nicht, oder die entstehende Curve war länger und niedriger als die vorhergehende. Ausspülen mit Kochsalzblut bedingte eine Erholung, welche wieder Contractionen auf Reiz zuliess, wo sie verschwunden gewesen waren.

Hierbei wurde noch etwas besonderes beobachtet. Während nämlich das Herz vorher auf Reize blos eine Contraction gemacht hatte, traten nach der Durchleitung der $\mathrm{Na}_{2} \mathrm{CO}_{3}$-lösung oft selbstständige Pulsationen auf.

Aus den angeführten Versuchen lässt sich wohl zunächst der 
Versuche über einige physiologische Wirkungen des Natriumcarbonates. 37

Schluss ziehen, dass die Wirkung des Natriumcarbonates auf den Ventrikel in einer Schädigung seiner Substanz liegt, und nicht auf eine Reizung hemmender Nerven zurïckzuführen ist. Für diese Deutung der erhaltenen Resultate spricht auch noch das gesonderte Verhalten der rechten und linken Ventrikelhälfte, wenn wenig Lösung gegeben war, wobei sich die Injection in der zunächst betroffenen linken Ventrikelhälfte allein und örtlich wirksam zeigte, während sie doch Stillstand des ganzen Herzens hätte bewirken miissen, wenn ihre Wirkung die Hemmungsnerven betroffen hätte. Für die Wirkung des kohlensauren Natrons auf das ganze Herz lassen sich Grüde noch nicht anführen, und es muss erst noch besonders untersucht werden, ob die eintretende Verlangsamung der Pulse in Reizung hemmender oder Lähmung beschleunigender Nerven ihren Grund hat. Ferner sind die Versuche auch noch an Warmblitern auszuführen.

Zum Schluss kann ich nicht unterlassen, dem Herrn Professor Bernstein für die grosse Freundlichkeit, mit der er diese Arbeit geleitet und überwacht hat, meinen besten Dank hiermit abzustatten.

\section{Erlänterungen zu den beigegebenen Curvenabschnitten anf Tafel II.}

Die beigefügten Längen bedeuten die Länge einer Minute. Die Curven sind von rechts nach links zu lesen.

Nro. I. a) Versuch am Kochsalzfrosch.

$I=$ Injection von $0,3 \mathrm{ccm} 5 \%$ iger Lösung.

b) Ausschnitt nach Verlauf von 20 Minuten gegeben, um die eingetretene Verlangsamung zu zeigen; einzelne Doppelwellen zeigen sich.

II. Versuch desgl.

$\mathrm{I}_{1}=$ Injection von $0,5 \mathrm{ccm} 5 \%$ iger Lösung. Die Verlangsamung tritt sofort ein.

$\mathrm{I}_{2}=$ Kochsalznachspülen, wobei wohl noch ein Rest der ersten Injection in's Herz kommt.

"III. Versuch desgl.

Die Elevationen haben im absteigenden Theil mehrere kleinere

Wellen aufgesetzt. 50 Minuten nach der ersten Injection. 
Nro. IV. Versuch desgl.

a) Abwechselnd Pulse von grösserem und kleinerem Umfang. 8 Minuten nach der ersten Injection.

b) Dieselben 27 Minuten nach der ersten Injection.

V. Versuch mit Herzkanüle und abgeschnittenem Ventrikel, bei welchem der Ventrikel unter Einfluss des Natriumcarbonates nicht contractionsfähig ist.

$\left.\begin{array}{l}\mathrm{S}=\text { Schliessung } \\ \mathrm{O}=\text { Oeffnung }\end{array}\right\}$ des primären Stromes.

$\mathrm{R}=$ Rollenabstand.

$\mathrm{Na}_{2} \mathrm{CO}_{3}=$ Durchwaschung mit kohlensaurem Natronblut.

$\mathrm{NaCl}=$ Kochsalzblut durchgespült.

VI. Versuch desgl., bei welchem die Contractionen flach und gedehnt wurden. Die Bezeichnungen wie vorher.

\section{Ueber das Paraglobulin.}

Von

\section{Olof Hammarsten.}

\section{Zweiter Abschnitt.}

Eine ungleiche Löslichkeit, resp. Fällbarkeit, wird bekanntlich als ein sehr wichtiger Unterschied zwischen den verschiedenen Eiweissstoffen betrachtet, und man hat dabei vor Allem dem Verhalten dieser Stoffe zu Neutralsalzen ein grosses Gewicht beigelegt. Unter solchen Umständen war es nicht nur von Interesse, das Verhalten des Paraglobulins zu Neutralsalzen im Allgemeinen zu prïfen, sondern es musste - mit Rücksicht auf die Fibrinfrage und das von mir zur Reindarstellung des Fibrinogens eingeschlagene Verfahren - für mich von besonderer Wichtigkeit sein, das Verhalten dieses Eiweissstoffes zu Kochsalz einer eingehenderen Prifung zu unterwerfen. Ich habe desshalb auch zu meinen Versuchen fast ausschliesslich dieses Salz verwendet und ich werde nun in diesem 2. Abschnitte meiner Abhandlung zuerst iber diejenigen Versuchsergebnisse berichten, welche auf das Verhalten des Para- 


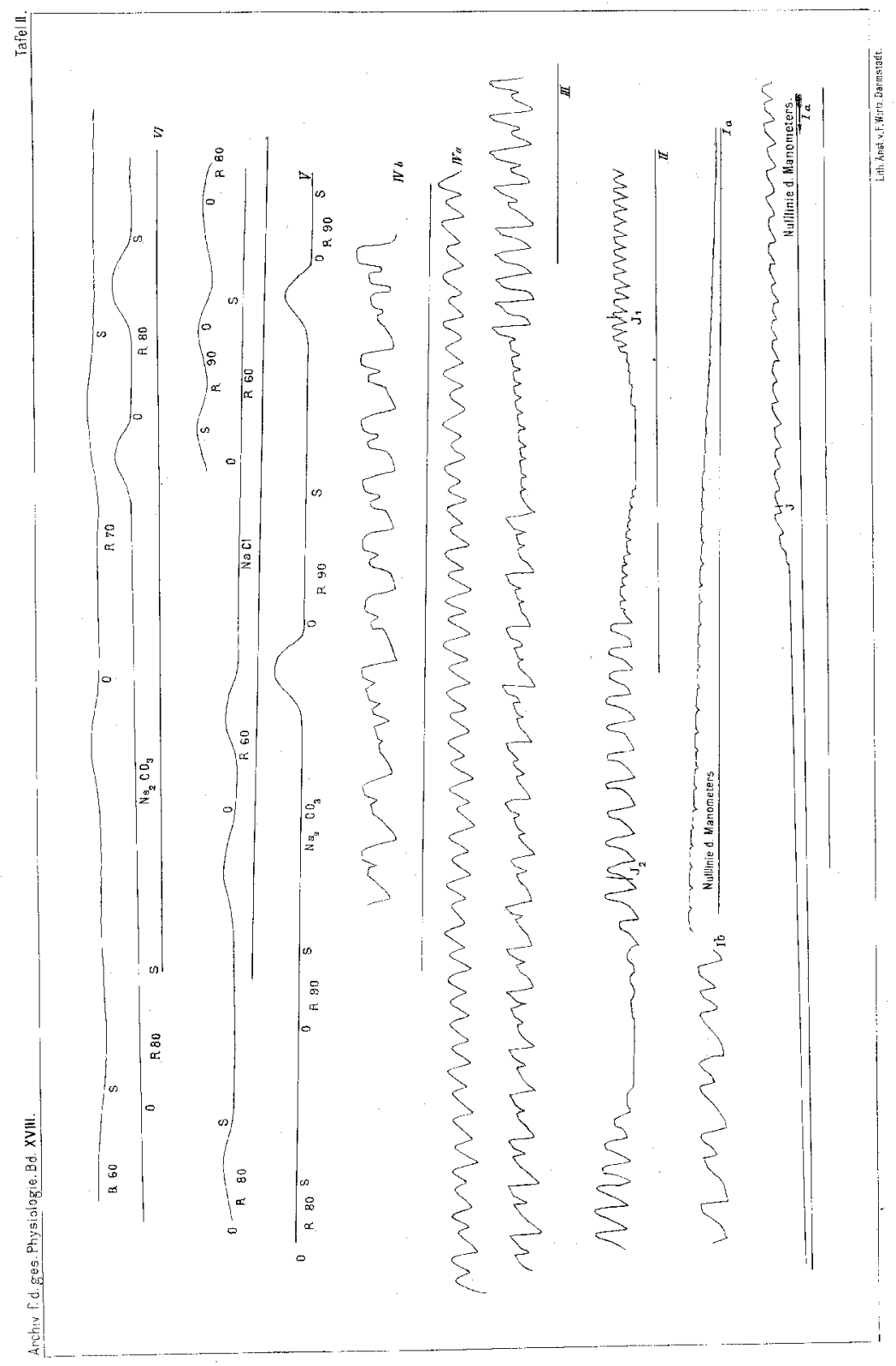

